Article

\title{
Simulation and Feature Extraction of the Dynamic Electromagnetic Scattering of a Hypersonic Vehicle Covered with Plasma Sheath
}

\author{
Zheng Bian $\mathbb{D}^{\mathbb{D}}$, Jiangting $\mathrm{Li} * \mathbb{D}$ and Lixin Guo \\ School of Physics and Photoelectric Engineering, Xidian University, Xi'an 710071, China; \\ zbian@stu.xidian.edu.cn (Z.B.); lxguo@xidian.edu.cn (L.G.) \\ * Correspondence: jtli@xidian.edu.cn; Tel.: +86-189-9136-3650
}

Received: 19 July 2020; Accepted: 19 August 2020; Published: 24 August 2020

\begin{abstract}
Based on the detection platform of space-borne radar (SBR), the dynamic electromagnetic (EM) scattering characteristics of a hypersonic vehicle covered with plasma sheath are calculated and analyzed by the physical optics (PO) method. Using the angle-time conversion method, the dynamic radar cross section (DRCS) of the vehicle in horizontal flying path and different flying states is solved in the range of L-band to X-band. The relevant effective statistical features are extracted from the time-scale matrix of DRCS based on continuous wavelet transform (CWT). The numerical results show that: At different flying altitudes, the backscattering radar cross section (BRCS) of the hypersonic vehicle covered with plasma sheath has irregular fluctuation and large range reduction in the head region of the vehicle. Generally, the higher the incident EM wave frequency is, the more detailed the fluctuation characteristics of the DRCS can be reflected. Within the same radar illuminate angle range, the time consumed by the vehicle DRCS is inversely proportional to the flying height and flying speed and is directly proportional to the detection altitude of the SBR. Besides, the different kinds of statistical eigenvalues extracted from DRCS will play an important role in hypersonic targets classification and recognition.
\end{abstract}

Keywords: hypersonic vehicle; plasma sheath; physical optics; dynamic radar cross section; continuous wavelet transform

\section{Introduction}

Detection and recognition of hypersonic vehicle by radar remote sensing involves the study of dynamic EM scattering of metal targets combined with the plasma flow field [1,2]; especially without considering the influence of near-space clutter and noise, how to obtain reliable DRCS data of hypersonic target becomes more important. We can get different DRCS data according to different flying states of the target spacecraft and establish a complete classification and recognition database. However, when the aircraft is flying at ultra-high speed in near space, the gas molecules around the vehicle will be excited to ionize, and the outer surface of the vehicle will be covered with a thick layer of plasma, which is called "plasma sheath" in the field of radar remote sensing [3,4]. The plasma sheath absorbs and attenuates the detection radar wave emitted by the SBR, and even makes the EM wave deviate from the original propagation track, resulting in irregular and disordered changes of the scattered signals received by the radar, and thus the main information of the target is lost, which brings great challenges to radar remote sensing, tracking and identifying hypersonic targets [5-8].

In addition, the detection frequency range of the active space observation radar is mainly concentrated in the $\mathrm{L}-\mathrm{X}$ band, and the main detection method is to emit multi-frequency continuous plane EM waves. Most EM scattering technology research does not consider the use of pulse compression [9]. Besides, compared with small targets such as hypersonic vehicle, most existing 
space observation radars are low-resolution radars. Therefore, it is of great practical significance to explore and study the dynamic EM scattering and feature extraction of hypersonic vehicles in the low-resolution radar system [10,11].

At present, the main research targets in hypersonic recognition field can be divided into RAM-C and HTV-2 vehicles, both of which are electrically large targets. NASA has carried out experimental research on RAM-C blunt cone reentry vehicle and published a large number of reliable data with extremely high value $[12,13]$, but the cost is very expensive. Nowadays, with the development of computer technology and some reliable EM numerical methods, the EM simulation technology has gradually come into people's vision. In more cases, the real experiments on site are gradually replaced by computer simulation technology. In recent years, many research reports have studied the propagation and scattering of EM waves at different frequency bands in the plasma sheath by using the finite-difference time-domain (FDTD) method [14-17], the discontinuous Galerkin time-domain method (DGTD) [18], the method of moments (MOM) based on the volume-surface integral equations (VSIE) $[19,20]$, the physical optics (PO) method [21-23] based on the inhomogeneity zonal medium model (IZMM) [24] and the Monte Carlo method [25]. On the other hand, some research work is devoted to the measurement and calculation of the DRCS of the target model and the detection and recognition of simple unmanned aerial vehicles (UAVs), fighters and satellites with different physical shape characteristics [26-30]. However, to the authors' knowledge, there are few reports on the dynamic scattering characteristics of hypersonic vehicles flying in near space based on the spaceborne detection platform, and the interaction between EM wave and plasma sheath is not involved.

In general, an important part of hypersonic targets recognition for SBR is to extract the effective statistical features of the DRCS; there are mainly two methods: (1) extract mathematical features directly from time domain, such as extracting cyclic distribution features of DRCS time series or focusing on the establishment and solution of probability density function (PDF) and cumulative distribution function (CDF) [31,32]; and (2) extract features from transform domain (Fourier transform, wavelet transform, Merlin transform, etc.) [33,34]. Since wavelet transform does not introduce the "cross term" in the time-frequency plane as with the Wigner-Ville transform, and due to the introduction of the concept of multi-scale, the wavelet transform has different resolution at different positions in the time-scale (frequency) plane, which makes the wavelet transform have good local properties in both time domain and frequency domain [35].

In this paper, the parallel PO method is used to establish a more reliable theoretical model to simulate and analyze the dynamic EM scattering characteristics of hypersonic vehicles. To highlight the difference of hypersonic targets in different flying states and reduce the feature dimension, CWT is first needed to extract the features from the DRCS time series of the vehicle, and then the effective statistical features are extracted. The focus of the paper is to provide a database for accurate comparison and reference for SBR remote sensing, detection and recognition of hypersonic targets. The main contributions are as follows:

1. Based on the flow field data of a hypersonic vehicle covered with plasma sheath, the EM scattering characteristics of target spacecraft at different flying altitudes are calculated and analyzed. On this basis, the horizontal flying path is established; the angle-time conversion method is used to convert the BRCS of the target spacecraft into the DRCS; and the relevant influence of target spacecraft at different flying altitudes, flying speeds and the detected altitude of the space-borne radar on the DRCS is studied. Finally, a hypersonic target tracking and identification database based on space-borne radar in near space is established.

2. Using the continuous wavelet transform (CWT), the features of DRCS of hypersonic vehicle with different flying states are extracted in the time-scale matrix, and the 10 effective and stable statistical features extracted are taken as the identification features. This conclusion lays a data foundation for space-borne radar to recognize hypersonic targets.

The paper is arranged as follows. In Section 2, the PO method based on the multilayer lossy medium model is briefly introduced, and the angle-time conversion method is used to convert the 
BRCS of hypersonic vehicles in different flying states into the DRCS. Section 3 calculates and analyzes the BRCS of the target spacecraft at different flying altitudes. On this basis, the influence of the flying altitude, flying speed and space-borne radar detection altitude on DRCS is discussed, and the DRCS database for hypersonic vehicles is established. In Section 4, wavelet transform is carried out for the DRCS observation sequence of the target spacecraft, and effective statistical eigenvalues are extracted from the time-scale matrix. Section 5 summarizes the paper and draws some useful conclusions.

\section{Modelling and Methods Description}

\subsection{Building the Hypersonic Target Model}

In this paper, the hypersonic target is modeled by referring to NASA RAM C-II vehicle with rotationally symmetric structure [13]. Figure 1 shows the hypersonic vehicle physical model. The radius of the ball head is $\mathrm{R}=0.1524 \mathrm{~m}$, the total length of the body is $\mathrm{L}=0.1524+1.295=1.4474 \mathrm{~m}$ and the half cone angle is $\alpha=9^{\circ}$. The incident wave electric field is polarized along the X-axis (namely, VV polarization: electric field direction parallel to the XOZ plane (page plane) and perpendicular to the incident direction; all the calculation results of EM scattering in the following sections adopt this polarization mode). The plane EM wave incident along the direction of the head of the blunt cone is the same as the positive direction of the Z-axis.

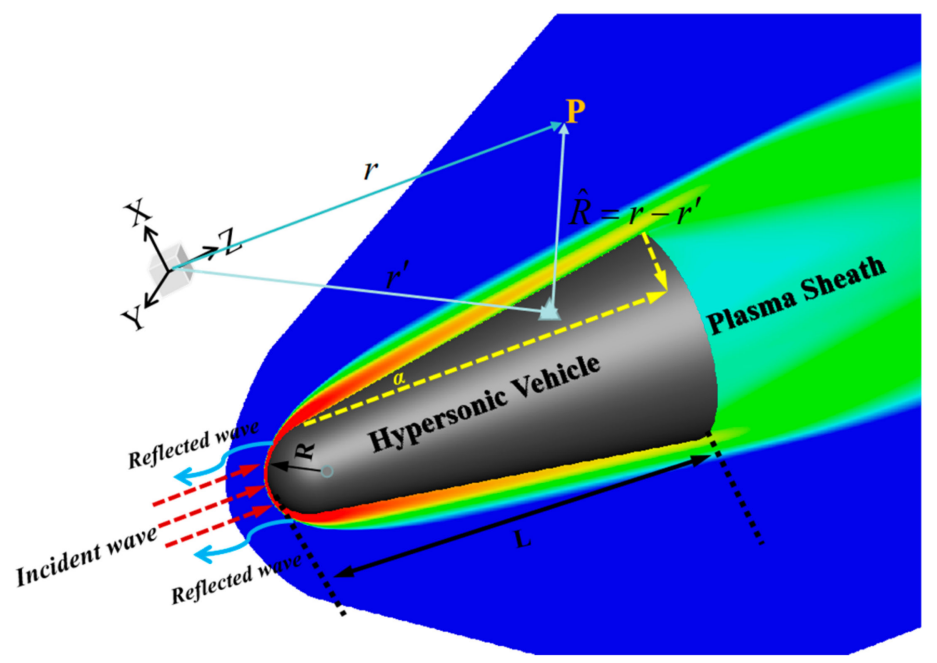

Figure 1. Geometric structure of hypersonic vehicle, $\mathrm{R}=0.1524 \mathrm{~m}, \mathrm{~L}=1.4474 \mathrm{~m}$ and $\alpha=9$

In this paper, based on the electrically large scale model of hypersonic vehicle and considering the main frequency range of spaceborne radar detection (L-band to X-band), the PO method based on the inhomogeneous zonal medium model (IZMM) is used to analyze the dynamic EM scattering of hypersonic vehicle [24]. In particular, the plasma fluid field data of the hypersonic vehicle is taken from the hypersonic vehicle plasma sheath and EM characteristics data manual [36].

\subsection{Physical Optics (PO) Method Based on the Multilayer Lossy Medium Model (MLMM)}

In this study, the BRCS of a hypersonic vehicle is calculated by using the PO method based on the Stratton-Chu scattering field integral equation. Here, the dielectric coefficient of the plasma sheath can be expressed by the Drude model [37] and we solve the collision frequency based on the piecewise calculation method proposed in [38]. According to Stratton-Chu equation and the geometric structure diagram of the hypersonic vehicle in Figure 1, after far-field approximation processing in the case 
of an incident plane EM wave, the electric and magnetic scattering fields of external point $\mathrm{P}$ can be calculated by Equations (1) and (2) [21]:

$$
\begin{aligned}
\mathbf{E}^{\mathrm{S}} & =\frac{-\mathrm{jk}}{4 \pi} \frac{\exp (-\mathrm{jkr})}{\mathrm{r}} \iint_{\mathrm{S}_{1}} \hat{\mathrm{R}} \times\left[\hat{\mathrm{n}} \times \mathbf{E}^{\mathrm{T}}-\eta \hat{\mathrm{R}} \times\left(\hat{\mathrm{n}} \times \mathbf{H}^{\mathrm{T}}\right)\right] \exp \left(\mathrm{jk} \hat{\mathrm{R}} \cdot \mathbf{r}^{\prime}\right) \mathrm{ds}^{\prime} \\
\mathbf{H}^{\mathrm{S}} & =\frac{-\mathrm{jk}}{4 \pi} \frac{\exp (-\mathrm{jkr})}{\mathrm{r}} \iint_{\mathrm{S}_{1}} \hat{\mathrm{R}} \times\left[\hat{\mathrm{n}} \times \mathbf{H}^{\mathrm{T}}+\frac{1}{\eta} \hat{\mathrm{R}} \times\left(\hat{\mathrm{n}} \times \mathbf{E}^{\mathrm{T}}\right)\right] \exp \left(\mathrm{jk} \hat{\mathrm{R}} \cdot \mathbf{r}^{\prime}\right) \mathrm{ds}^{\prime}
\end{aligned}
$$

among them,

$$
\begin{gathered}
\hat{\mathrm{n}} \times \mathbf{E}^{\mathrm{T}}=\left(1+\mathrm{R}_{\perp}\right) \mathrm{E}_{\perp}\left(\hat{\mathrm{n}} \times \hat{\mathrm{e}}_{\perp}\right)-\left(1-\mathrm{R}_{/ /}\right) \mathrm{E}_{/ / / \cos \theta_{\mathrm{i}} \hat{\mathrm{e}}_{\perp}} \\
\hat{\mathrm{n}} \times \mathbf{H}^{\mathrm{T}}=\frac{1}{\eta}\left[\left(1-\mathrm{R}_{\perp}\right) \mathrm{E}_{\perp} \cos \theta_{\mathrm{i}} \hat{\mathrm{e}}_{\perp}+\left(1+\mathrm{R}_{/ /}\right) \mathrm{E} / /\left(\hat{\mathrm{n}} \times \hat{\mathrm{e}}_{\perp}\right)\right]
\end{gathered}
$$

where $\mathrm{S}_{1}$ represents the bright area of the scatterer; $\mathbf{E}^{\mathrm{T}}$ and $\mathbf{H}^{\mathrm{T}}$ are the total electric field and the total magnetic field on the boundary, respectively; $k$ and $\eta$ are the propagation constant in free space and the intrinsic impedance, respectively; $\hat{R}$ is the unit vector of the scattered wave; $\mathbf{r}^{\prime}$ is the unit vector of the position of the scatterer unit; $\mathbf{r}$ is the position vector of point $\mathrm{P}$ (as shown in Figure 1); and $\mathbf{r}^{\prime}=\left|\overrightarrow{\mathrm{r}^{\prime}}\right|$ and $\mathbf{r}=|\vec{r}|$. $\hat{n}$ is the normal unit vector of the surface element at $\mathbf{r}^{\prime}$. In addition, $\theta_{\mathrm{i}}$ is the incident angle of the plane EM wave, $\hat{e}_{\perp}$ vector is the polarization direction of the incident and reflected electric fields perpendicular to the incident plane and $\mathrm{E}_{\perp}$ and $\mathrm{E}_{/ /}$are the field components of incident electric field in $\hat{e}_{\perp}$ and $\hat{e}_{/ /}^{i}$ directions, respectively.

$R_{\perp}$ and $R_{/ /}$are the reflection coefficients of the dielectric surface at vertical and parallel polarization, respectively. Here, the Stepping method is adopted to solve it [22]. In addition, the incident EM wave is usually approximated by the plane EM wave:

$$
\begin{aligned}
\mathbf{E}^{\mathrm{i}}\left(\mathbf{r}^{\prime}\right) & =\hat{\mathrm{e}}_{\mathrm{i}} \mathrm{E}_{0} \mathrm{e}^{-\mathrm{j} \mathrm{k}_{\mathrm{i}} \cdot \mathrm{r}^{\prime}} \\
\mathbf{H}^{\mathrm{i}}\left(\mathbf{r}^{\prime}\right) & =\frac{1}{\eta} \hat{\mathrm{k}}_{\mathrm{i}} \times \mathrm{E}^{\mathrm{i}}\left(\mathbf{r}^{\prime}\right)
\end{aligned}
$$

According to Equations (1)-(6), under far-field scattering, the computational equation of the BRCS of a hypersonic vehicle is as follows:

$$
\sigma_{\text {BRCS }}=\lim _{\mathrm{R} \rightarrow \infty} 4 \pi \mathrm{R}^{2} \frac{\left|\mathbf{E}^{\mathrm{S}}\right|^{2}}{\left|\mathbf{E}^{\mathrm{i}}\right|^{2}}
$$

\subsection{Angle-Time Conversion Method Based on Horizontal Flying Path}

Dynamic RCS (DRCS) can show not only the scattering ability of the target spacecraft to the EM wave radiated by radar, but also the flying altitude, flying speed, radar line-of-sight angle and other feature information of the target spacecraft. In this paper, the hypersonic vehicle is set as the maneuver mode of side station level flying (i.e., the lift of the vehicle is equal to gravity and the thrust is equal to the drag); in the process of side station level flying, the azimuth angle of radar line of sight to the target remains unchanged (default the included angle is $0^{\circ}$ in calculation), the roll angle is $0^{\circ}$ and only the pitch angle $\theta$ is changed. This section describes the use of a quasi-static method to obtain the dynamic RCS sequence of the target spacecraft, namely the hypersonic vehicle RAM C-II as the research object. In addition, the target spacecraft is researched along a horizontal air route path when flying at different altitudes and speeds. The dynamic RCS characteristics along with the change of time and frequency response characteristics of radar detection and identification of the hypersonic vehicle are the basis of the simulation. 
Here, the angle-time conversion method is used to solve the dynamic RCS sequence of the vehicle. The flying time of the hypersonic target varies unevenly with the unit angle during the distance from the space-borne radar. As shown in Figure 2, $\mathrm{H}=200,000 \mathrm{~m}$ is the height from the ground of the space-borne radar mounted on the non-synchronous satellite, $\mathrm{H}_{1}$ and $\mathrm{H}_{2}$ are the different flying heights of the hypersonic vehicle and $\mathrm{H}_{0}$ is the shortest vertical distance between the vehicle and the space-borne radar.

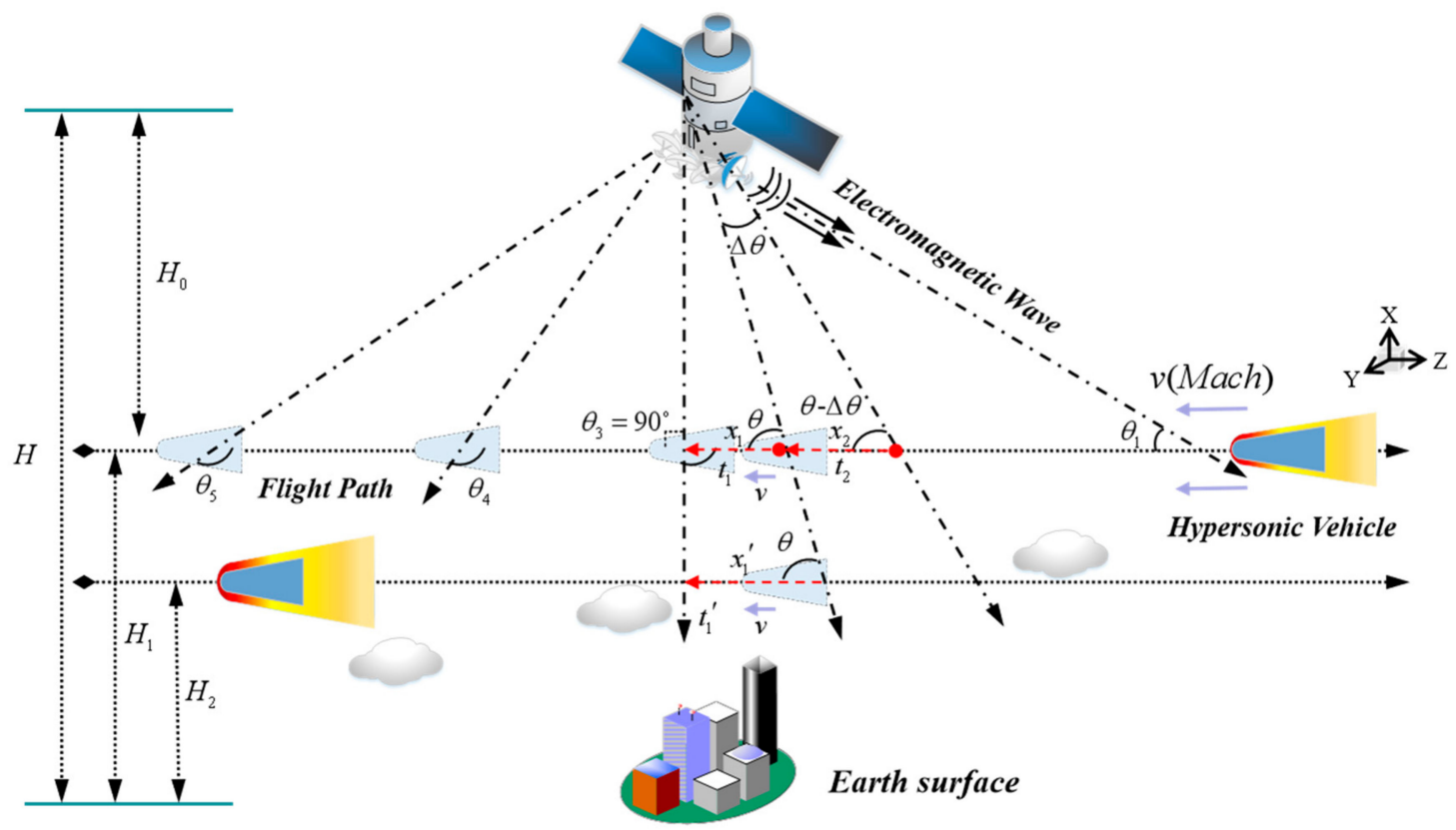

Figure 2. Hypersonic vehicle flying path and DRCS observation diagram.

This model calculates the accumulated time of hypersonic vehicle at different positions when the pitch angle between the vehicle axis and the radar illuminate direction varies from $2.5^{\circ} \leq \theta \leq 177.5^{\circ}$; that is, when the angle between the incident direction of the space-borne radar EM wave and the axis of the target spacecraft is $2.5^{\circ}$, the initial time at this time is recorded as $0 \mathrm{~s}$. Here, the first half of the flying path of the vehicle is taken as the calculation interval, the initial value is $\theta=89.5^{\circ}$, the change in unit angle is $\Delta \theta=1^{\circ}$ and the range of change times of the angle in the first half is $0 \leq \mathrm{n} \leq 87$. Then, the interval in which the angle changes in the first half flying of the hypersonic vehicle is:

$$
2.5^{\circ} \leq \theta-\mathrm{n} \Delta \theta \leq 89.5^{\circ}
$$

Further, if $\mathrm{v}$ is the average speed of the vehicle on the horizontal flying path, then

$$
\begin{gathered}
\mathrm{x}_{1}=\frac{\mathrm{H}_{0}}{\tan (\theta)}, \mathrm{t}_{1}=\frac{\mathrm{x}_{1}}{\mathrm{v}} \\
\mathrm{x}_{2}=\frac{\mathrm{H}_{0}}{\tan (\theta-\Delta \theta)}-\mathrm{x}_{1}, \mathrm{t}_{2}=\frac{\mathrm{x}_{2}}{\mathrm{v}} \\
\mathrm{x}_{3}=\frac{\mathrm{H}_{0}}{\tan (\theta-2 \Delta \theta)}-\mathrm{x}_{2}, \mathrm{t}_{3}=\frac{\mathrm{x}_{3}}{\mathrm{v}} \\
\mathrm{x}_{\mathrm{n}+1}=\frac{\mathrm{H}_{0}}{\tan (\theta-\mathrm{n} \Delta \theta)}-\mathrm{x}_{\mathrm{n}}, \mathrm{t}_{\mathrm{n}+1}=\frac{\mathrm{x}_{\mathrm{n}+1}}{\mathrm{v}}
\end{gathered}
$$


For the first half of the flying path, the cumulative time of the vehicle from infinite distance to each fixed angle is:

$$
\mathrm{T}_{\mathrm{n}_{\max }+1}=\sum_{\mathrm{n}=0}^{\mathrm{n}_{\max }} \mathrm{t}_{88-\mathrm{n}}\left(0 \leq \mathrm{n}_{\max } \leq 87\right)
$$

Similarly, in the second half of the flying path, the time taken for the vehicle to fly to each fixed angle is symmetrical to that of the first half. Then, the vehicle flies from the vertical shortest distance point $\left(\theta=90^{\circ}\right)$ of the space-borne radar to the infinite distance. The accumulated time at each fixed angle is:

$$
\mathrm{T}_{\mathrm{m}_{\max }}=\sum_{\mathrm{m}=89}^{\mathrm{m}_{\max }}\left(\mathrm{T}_{\mathrm{m}-1}+\mathrm{t}_{\mathrm{m}-88}\right)\left(89 \leq \mathrm{m}_{\max } \leq 176\right)
$$

where $\mathrm{m}$ is the number of times the angle changes in the second half, and the accumulated time in the first and second half is the total time taken by the hypersonic vehicle to follow the horizontal flying path at a fixed altitude. Therefore, the accumulated time taken by the vehicle to reach each radar incident angle on the horizontal flying path can be calculated.

In addition, when the hypersonic vehicle flies to a fixed position along the flying path, in other words, when it reaches a certain angle $\theta$ between the flying direction and the incident direction of space-borne radar incident, it takes different times for an vehicle at different altitudes to reach the same position at the same speed v. As shown in Figure 2, when the flying altitude of the vehicle is $\mathrm{H}_{2}<\mathrm{H}_{1}$, in both cases, the flying direction of the vehicle and the detection direction of space-borne radar are at the same included angle $\theta$, the horizontal distance $x_{1}^{\prime}>x_{1}$, namely, $t_{1}^{\prime}>t_{1}$. In other words, when the hypersonic vehicle is flying at different altitudes, the accumulated flying time is different when the target spacecraft and the radar irradiation direction form an included angle. The lower is the flying altitude, the longer is the required flying time.

To sum up, this paper takes hypersonic vehicle as the research object and simulates the dynamic EM scattering characteristics of its flying on a set path. The specific steps are as follows:

1. Obtain the plasma flow field data of hypersonic vehicles in different flying states and establish a BRCS EM scattering research model for hypersonic vehicles.

2. The physical optics (PO) method based on IZMM is used to simulate the EM scattering characteristics of hypersonic vehicles at different radar line-of-sight angles.

3. According to the performance parameters of the vehicle and the basic theory of aerodynamics, the flying path of the hypersonic target is set, and the angle-time conversion method is used to convert the BRCS of the vehicle into the DRCS.

\section{Simulation Results and Discussion}

\subsection{Influence of Radar Observation Angle on EM Scattering Properties of Hypersonic Vehicle}

This section calculates the BRCS of hypersonic vehicle in the range of $0.5^{\circ}$ to $179.5^{\circ}$ at different flying altitudes. Physical modeling and EM simulation calculations were conducted on a PC with 8 GB RAM and eight 2.6 GHz Intel i7-6700 HQ CPUs. The number of surface subsections of the outer surface of the target aircraft varied according to the flying states of the vehicle covered with the plasma sheath. Table 1 lists the number of triangular patches that subdivide the geometric model (Taking 1/8 of $12 \mathrm{GHz}$ EM wavelength as the dividing criterion) and the time required to calculate the BRCS of the hypersonic targets at $20 \mathrm{Ma}$ and different flying altitudes.

Figure 3 shows the electron density distribution of hypersonic vehicle at different flying altitudes [36]. It can be found that, with the increase of flying altitude of the hypersonic target, the plasma sheath thickness around the vehicle becomes thinner gradually, and the electron density begins to decline. This is mainly because, as the air at high altitude is relatively thin, the air ionization is less, and the electron density of plasma coated around the vehicle surface is low. When the flying 
altitude drops from 70 to $30 \mathrm{~km}$, the low altitude atmosphere is relatively dense, the air on the vehicle surface has large ionization and the electron density of plasma increases gradually.

Table 1. Computational time of BRCS for selected flying states.

\begin{tabular}{ccc}
\hline Flying States & Total Number of Surface Elements & Total Computing Time \\
\hline $\mathbf{3 0} \mathbf{~ k m ~ 2 0 ~ M a}$ & $1,378,982$ & $2.01 \mathrm{~h}$ \\
$\mathbf{4 0} \mathbf{~ k m ~} \mathbf{2 0} \mathbf{~ M a}$ & $1,166,718$ & $1.70 \mathrm{~h}$ \\
$\mathbf{5 0} \mathbf{~ k m ~ 2 0 ~ M a}$ & $1,166,256$ & $1.70 \mathrm{~h}$ \\
$\mathbf{6 0} \mathbf{~ k m ~ 2 0 ~ M a}$ & $1,367,902$ & $1.92 \mathrm{~h}$ \\
$\mathbf{7 0} \mathbf{~ k m ~ 2 0} \mathbf{~ M a}$ & $1,406,762$ & $2.01 \mathrm{~h}$ \\
\hline
\end{tabular}

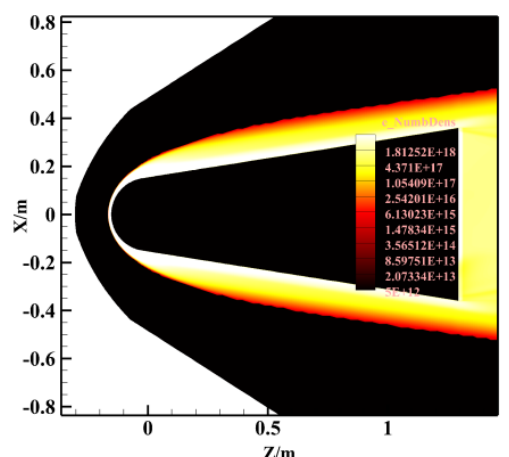

(a)

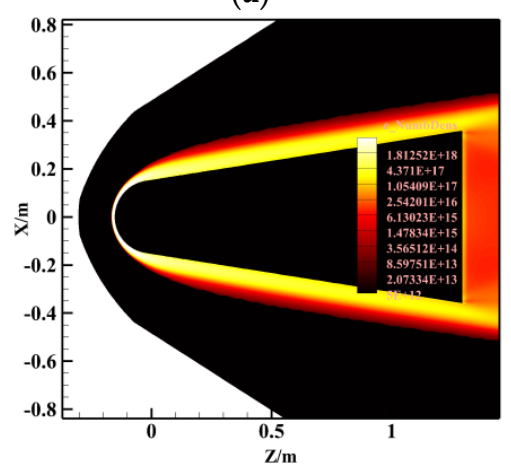

(d)

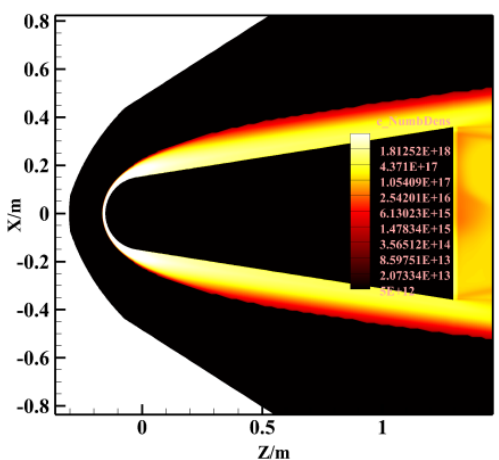

(b)

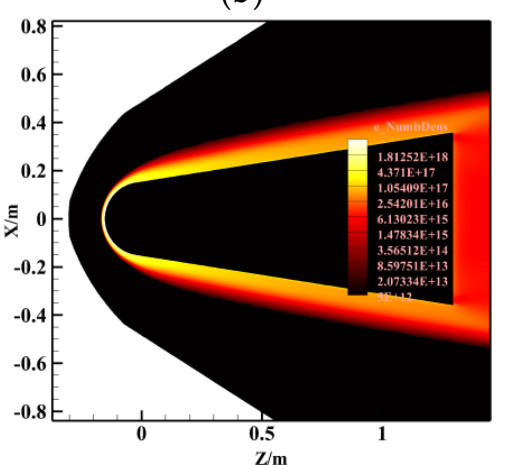

(e)

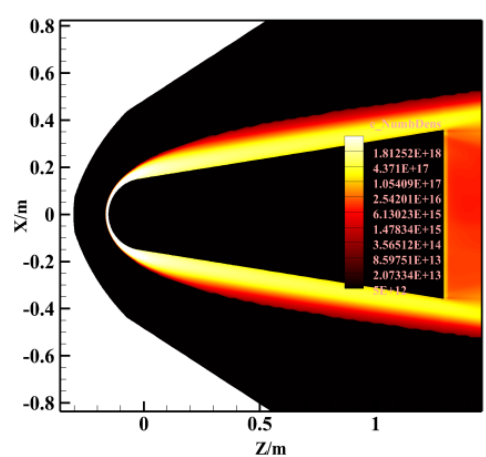

(c)

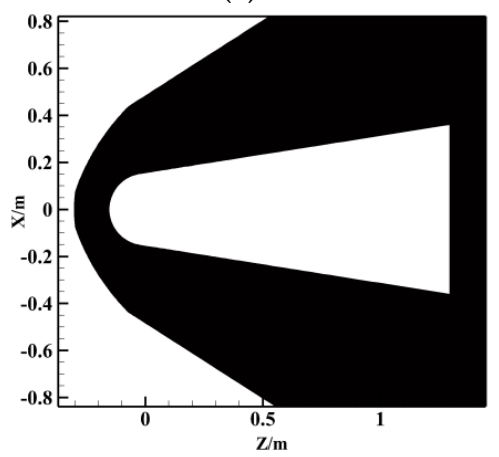

(f)

Figure 3. Electron density distribution field of the plasma sheath at: (a) $30 \mathrm{~km}, 20 \mathrm{Ma}$; (b) $40 \mathrm{~km}, 20 \mathrm{Ma}$; (c) $50 \mathrm{~km}, 20 \mathrm{Ma}$; (d) $60 \mathrm{~km}, 20 \mathrm{Ma}$; (e) $70 \mathrm{~km}, 20 \mathrm{Ma}$; and (f) $0 \mathrm{~km}, 0 \mathrm{Ma}$.

Figure 4 shows the BRCS comparison of hypersonic vehicles covered with plasma sheath at different flying altitudes. The EM wave of the detection radar is incident from the head of the blunt cone with an initial angle of $\theta=0^{\circ}$. When the incident angle is $\theta=81^{\circ}$ and $\theta=261^{\circ}$, the projection area of the blunt cone spacecraft along the direction of radar irradiation is the largest, and the BRCS reaches a maximum peak point at this point. Similarly, BRCS reaches a maximum value at the tail of the blunt cone.

Compared with the curve fluctuation of pure metal blunt cone BRCS, the existence of plasma sheath makes the BRCS of the hypersonic target have a large irregular fluctuation and attenuation in most of the incident angle directions, especially in the illuminate angle range of the vehicle head area. This is due to the high electron density of plasma in the head area of the vehicle; however, the sheath is very thin (as the plasma flow field distribution in Figure 3) and the plasma will produce large collision and absorption of the incident EM wave. In addition, as the flying altitude of the hypersonic target continues to decrease, the BRCS gradually decreases at all angles, especially at the incident angle of $81^{\circ}$. The peak value of the BRCS at the flying altitude of the vehicle at $70 \mathrm{~km}$ from the ground is larger than 
at the other four flying altitudes. Similarly, at an altitude of $30 \mathrm{~km}$, the BRCS of the hypersonic vehicle covered with a plasma sheath is reduced in almost all incident directions (see the red line in Figure 4).

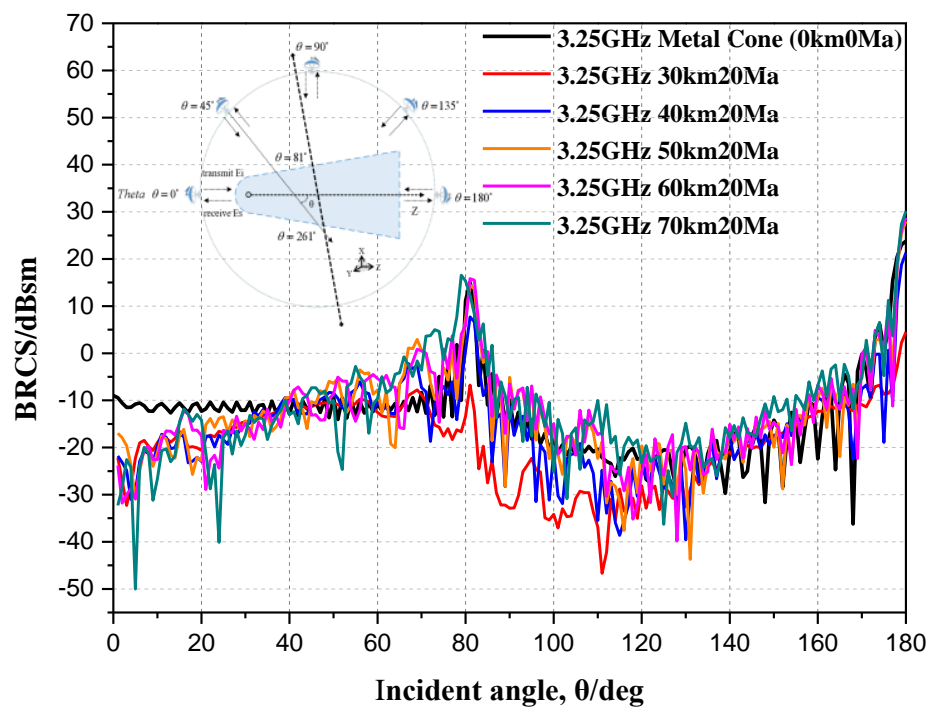

Figure 4. Variation of BRCS with flying altitude for hypersonic vehicle, when the incident wave frequency is $3.25 \mathrm{GHz}$ and the velocity is 20 Mach.

\subsection{Dynamic EM Scattering Analysis of Hypersonic Vehicle}

In this section, the space-borne radar is located on a non-synchronous satellite at a height of 200-300 km, and the EM wave bands irradiated by the detection radar are the L-X bands. We studied the effects of incident EM wave frequency, space-borne radar detection altitude, flying altitude and flying speed of the target spacecraft on the dynamic RCS time series of a hypersonic vehicle covered with a plasma sheath when the range of the angle between the target spacecraft and the radar irradiation direction is $2.5^{\circ} \leq \theta \leq 177.5^{\circ}$. Our objective was to establish a relatively complete dynamic RCS target recognition database for hypersonic vehicle of low-resolution radar. The simulation results are shown in Figure 5a-d.

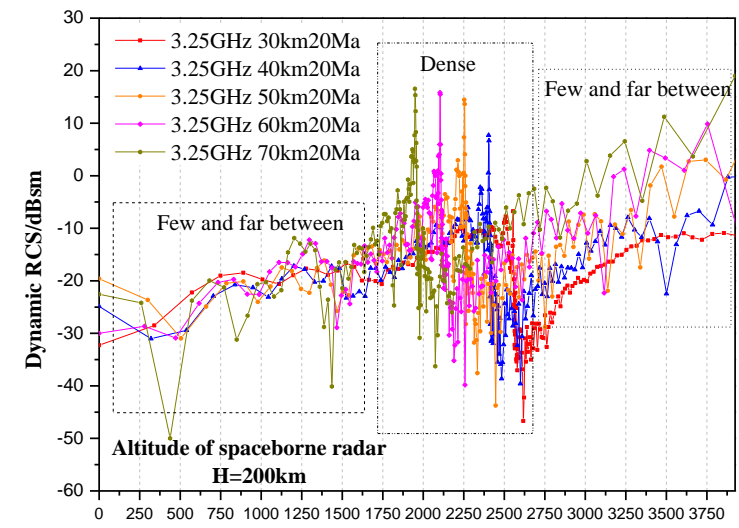

$\mathrm{T} / \mathrm{s}$

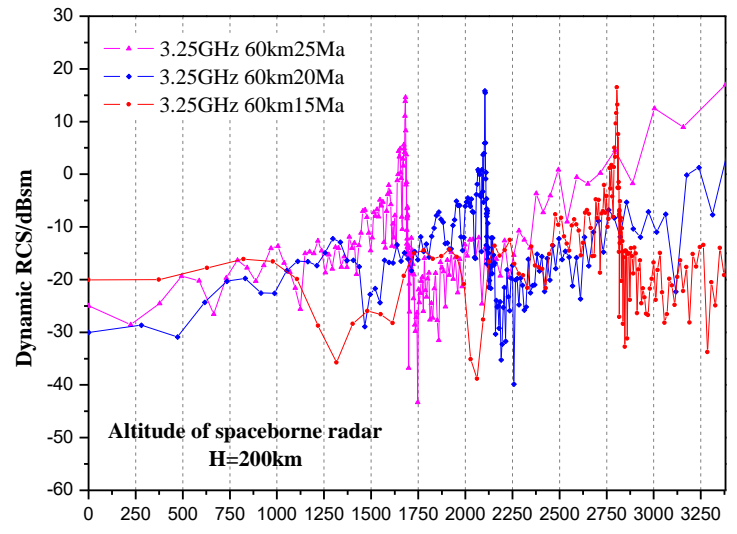

$\mathrm{T} / \mathrm{s}$

Figure 5. Cont. 


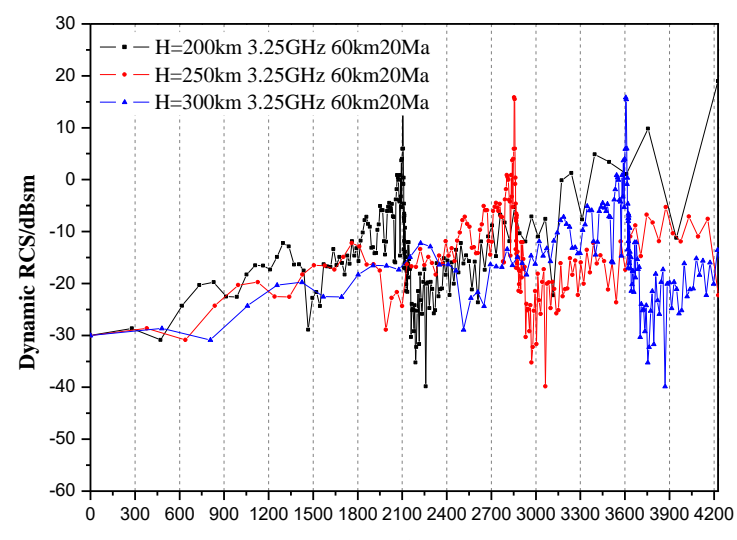

(c)

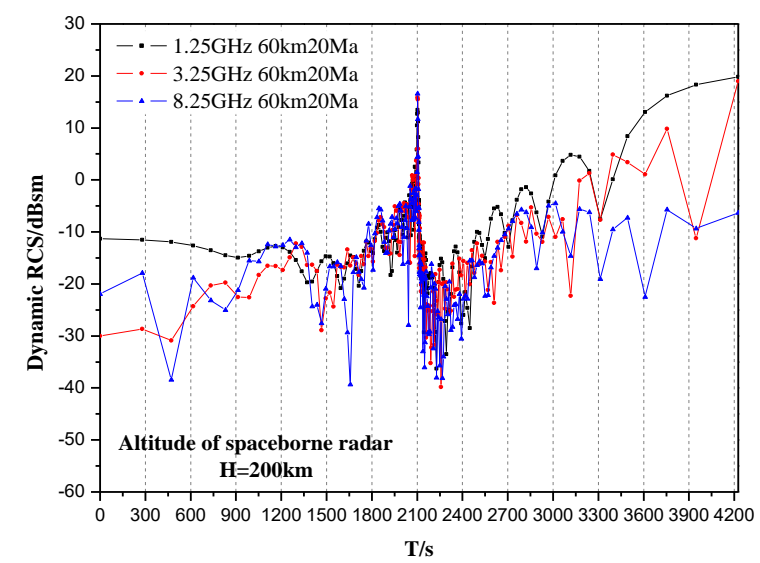

(d)

Figure 5. Dynamic RCS sequence comparison of the plasma sheath for hypersonic vehicle flying at different incident electromagnetic frequencies, space-borne radar detection heights, and flying states. (a) Space-borne radar at an altitude of $200 \mathrm{~km}$, incident EM wave frequency of $3.25 \mathrm{GHz}$ and the flying speed of the target spacecraft is 20 Mach; flying altitudes of 70, 60, 45 and $30 \mathrm{~km}$ are compared. (b) Space-borne radar at an altitude of $200 \mathrm{~km}$, incident electromagnetic wave frequency of $3.25 \mathrm{GHz}$ and the target spacecraft flying at an altitude of $60 \mathrm{~km}$; flying speeds of 15, 20 and 25 Mach are compared. (c) Incident EM wave frequency of $3.25 \mathrm{GHz}$, velocity and height are 20 Mach and $60 \mathrm{~km}$ above ground, respectively; altitudes of 200, 250 and $300 \mathrm{~km}$ are compared. (d) Space-borne radar at an altitude of $200 \mathrm{~km}$, velocity and height are $20 \mathrm{Mach}$ and $60 \mathrm{~km}$ above ground, respectively; frequencies of 1.25, 3.25 and $8.25 \mathrm{GHz}$ are compared.

The technical approach we follow is to convert the backscattering RCS of the target spacecraft with the change in radar incident angle into the dynamic RCS time series as a function of time. The dynamic RCS curve in Figure 5a clearly shows that, with the passage of time, for a target spacecraft flying at a constant speed along the horizontal path, when the distance between the space-borne radar and the target spacecraft changes from far to near and then to far, the fluctuations of the dynamic RCS curve change from gentle to violent and then to gentle. In addition, the distribution of scattering data points of the hypersonic target is from sparse to dense and then to sparse (as shown in Figure 5a, where the curve changes in the three dotted boxes).

When the hypersonic vehicle is located at a great distance from the space-borne radar, its dynamic RCS curve fluctuates gently, and the scattered data points on the curve are distributed sparsely. However, as the target spacecraft approaches the detection radar, until the vehicle flies directly below the space-borne radar (the line between the two centers is perpendicular to the horizontal flying path, i.e., $\theta=90^{\circ}$ ), the scattering points on the dynamic RCS curve are the densest with time, and the curve oscillations and jitters are the most intense. This is because the target vehicle is traveling at a constant speed, and, in the process of reducing the distance from the space-borne radar from far to near, the flying time varies unevenly with the unit angle. In other words, as the distance between the vehicle and the detection radar gradually shortens, the flying time of the vehicle gradually decreases when the target spacecraft is scanned by the detection radar at a unit angle. Similarly, while the hypersonic vehicle is flying away from the satellite-borne radar at constant speed, when the detection radar illuminates the target spacecraft and sweeps through the unit angle, the flying time of the vehicle gradually increases from short to long, and the fluctuation of the dynamic RCS curve gradually becomes gentle.

The numerical distribution and fluctuation change of the dynamic RCS curve are related to the flying altitude and flying speed. According to the flying path and the relative position of the space-borne radar and hypersonic vehicle, it can be concluded that, as shown in Figure $5 \mathrm{a}$, the higher is the flying altitude $\left(\mathrm{H}_{\mathrm{x}}=30 \mathrm{~km}, 40 \mathrm{~km}, 50 \mathrm{~km}, 60 \mathrm{~km}\right.$ and $\left.70 \mathrm{~km}\right)$, that is, the closer is the hypersonic vehicle to the space-borne radar, the shorter is the time required for the radar to sweep through the 
fuselage unit angle at the same flying Mach number $(v=20$ Mach). In other words, the faster does the dynamic RCS fluctuation of the target spacecraft change in the same time range, the shorter is the time for the curve to change from sparse to dense and then to sparse.

Similarly, as shown in Figure $5 b$, at the same flying altitude $\left(H_{x}=60 \mathrm{~km}\right)$, the faster is the hypersonic vehicle flying ( $\mathrm{v}=15$ Mach, 20 Mach and 25 Mach), the shorter is the time required for the radar to sweep through the fuselage unit angle, that is, the target dynamic RCS curve can oscillate to the highest peak point in a short time. For example, the target spacecraft flew at an altitude of $60 \mathrm{~km}$ and a speed of 25 Mach (as shown in Figure 5b, the pink curve) when the dynamic RCS curve of the target spacecraft reached the densest center point. That is, when the connection between the vehicle and the space-borne radar center is perpendicular to the horizontal flying path, the dynamic RCS curve of the blunt cone spacecraft with flying altitude of $60 \mathrm{~km}$ and flying speed of 15 Mach (red curve in Figure $5 b$ ) is still in the gentle and sparse area.

Next, we studied the change in the dynamic RCS of the target spacecraft when the space-borne radar flies at different altitudes $(\mathrm{H}=200 \mathrm{~km}, 250 \mathrm{~km}$ and $300 \mathrm{~km})$. In short, the variation rule of dynamic RCS is similar to changes in the flying altitude of the target spacecraft, which is to change the distance between the target spacecraft and the detection radar. The further is the distance between the target spacecraft and the space-borne radar, the longer it takes for the radar to sweep through the fuselage unit angle at the same flying speed ( $\mathrm{v}=20 \mathrm{Mach})$ and the slower is the dynamic RCS fluctuation of the target spacecraft in the same time range, as shown by the blue curve of the target dynamic RCS in Figure 5c.

Then, we studied the extent to which the characteristics of the law of the dynamic RCS sequence of the target spacecraft change with the incident EM wave frequency when the frequency range of the incident EM wave is in the L-X bands and the flying state is $60 \mathrm{~km}$ and $20 \mathrm{Mach}$, as shown in Figure $5 \mathrm{~d}$. An increase in the frequency of incident EM waves, which would be more obvious, highlights details of the features of the physical structure of the target spacecraft, compared with the target at the low-frequency $1.25 \mathrm{GHz}$ dynamic RCS sequence. When the incident EM wave frequency increases to $8.25 \mathrm{GHz}$, the dynamic RCS time series curve of the target fluctuates and oscillates more dramatically, and the peak point of the curve becomes sharper when $\theta=81^{\circ}$. In addition, EM waves at higher frequencies can partially or completely penetrate the plasma sheath covering the surface of the target spacecraft, and the energy attenuation is greater because EM waves have a longer propagation path inside the plasma. However, when the hypersonic vehicle covered with a plasma sheath is illuminated by the EM wave at a lower frequency, the EM wave cannot penetrate the plasma sheath, and most of the EM waves are reflected from the surface of the plasma sheath, resulting in the high value of the dynamic RCS curve of the target spacecraft in a large time range. Therefore, effectively reducing the frequency of EM waves emitted by space-borne radar would be more helpful for detecting and tracking a hypersonic vehicle covered with a plasma sheath.

Finally, we calculated the frequency of incident EM wave as $\mathrm{f}=8.25 \mathrm{GHz}$; the incident electric field and scattering electric field are in the same polarizations mode $(\mathrm{VV} / \mathrm{HH})$, keep the same flying altitude $(60 \mathrm{~km})$ and at the different flying speeds. The DRCS time series of hypersonic vehicle are shown in Figure $6 \mathrm{a}, \mathrm{b}$ (where the detection altitude of space-borne radar remains unchanged, $\mathrm{H}=200 \mathrm{~km}$ ).

Figure $6 \mathrm{a}, \mathrm{b}$ shows that the polarization mode of radar antenna transmitting and receiving EM wave does have an impact on the DRCS time series of the hypersonic targets, which is mainly reflected in the slight difference of DRCS curve waveform trend and oscillation mode. Although these changes are irregular and fuzzy, they do have certain impact on hypersonic targets recognition. In addition, for the two polarization modes $(\mathrm{VV} / \mathrm{HH})$, the peak values of DRCS time series at $\theta=81^{\circ}$ and $\theta=180^{\circ}$ have no change. 


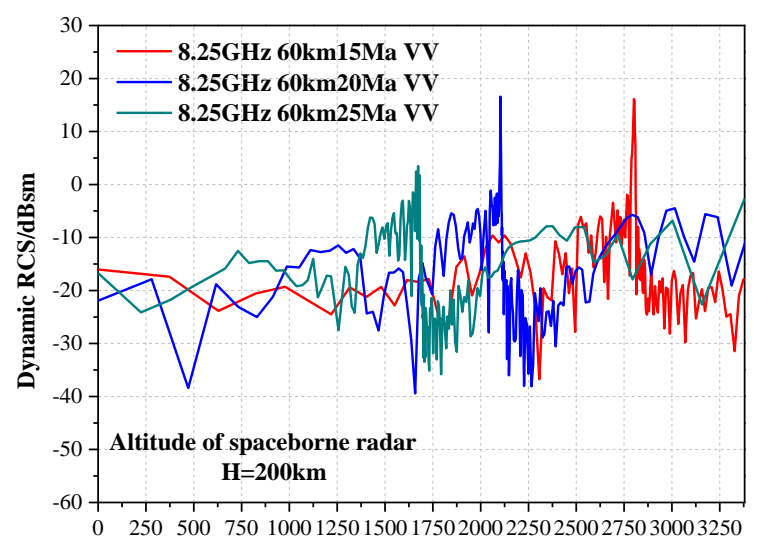

$\mathrm{T} / \mathrm{s}$

(a)

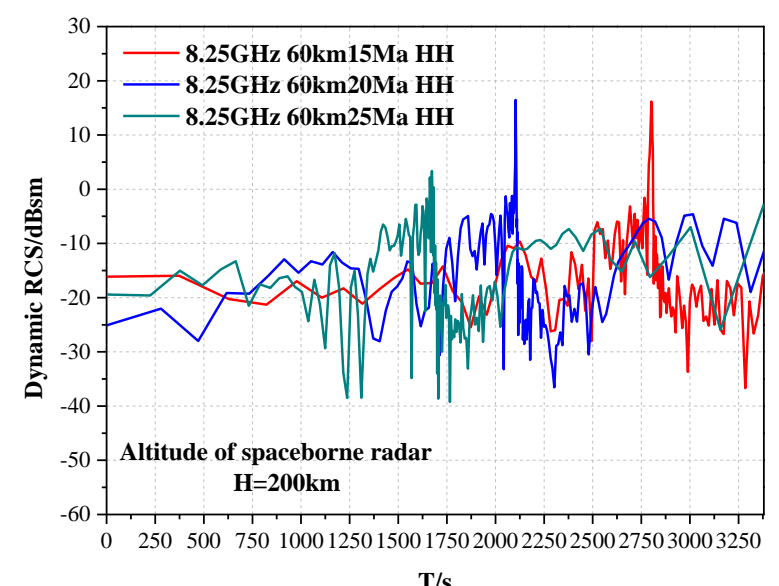

(b)

Figure 6. The DRCS time series of hypersonic target covered with plasma sheath at three different flying speeds. The flying altitude and incident EM wave frequency are taken to be $60 \mathrm{~km}$ and $8.25 \mathrm{GHz}$, respectively. (a) VV polarizations; and (b) $\mathrm{HH}$ polarizations.

\subsection{Mathematical Sensitivity Function of DRCS}

Due to the existence of plasma sheath and the maneuverability of hypersonic targets in space and time, the DRCS of target spacecrafts in different flying states tend to be quite different; thus, the change of DRCS is sensitive, which makes it possible for us to detect and identify hypersonic vehicles effectively. Based on the mathematical characteristics and distribution form of hypersonic targets DRCS, we use probability density function (PDF) and cumulative distribution function (CDF) to solve the probability distribution form of hypersonic targets DRCS coefficient under different flying altitudes, the same flying speed $(20 \mathrm{Ma})$ and the same flying altitude $(60 \mathrm{~km})$ and different flying speeds. The incident EM wave frequency is $\mathrm{f}=3.25 \mathrm{GHz}$, and the results are shown in Figure 7a-d. For the calculation results in Figure 7a,b, the uniform flying time period cut out by DRCS time series is [0 s-3916 s], similarly, for Figure 7c,d, the uniform flying time period cut out by DRCS time series is [0 s-3372 s] (see Figure 5a,b for the DRCS distribution in corresponding flying state of the hypersonic targets).

Figure 7a-d shows that the PDF and CDF curve distribution of the DRCS time series of hypersonic vehicle is extremely sensitive to the change of flying altitude and flying speed. The most important ones are the broadening of PDF curve and the change of probability peak (Figure 7a,c). In general, in the same detection period of space-borne radar (SBR), when the flying speed remains constant (20 Ma), the higher is the altitude of hypersonic vehicle, the more obvious is the broadening of PDF curve of DRCS, and the peak height of probability density is lower. This is because the higher is the flying altitude, the shorter is the relative distance between the target spacecraft and the SBR. In the same radar detection time range, the illuminate angle range of the radar scanning the hypersonic target is larger, and the distribution range of DRCS coefficient in the range [-60 dBsm, $30 \mathrm{dBsm}]$ becomes wider; accordingly, the peak value of DRCS coefficient distribution probability is lower. Similarly, at the same flying altitude $(60 \mathrm{~km})$, the larger is the flying Mach number, the greater is the broadening degree of PDF curve and the lower is the peak probability density, and the principle are the same.

In addition, different flying states of hypersonic targets affect seriously the slope of the CDF curve and the range of DRCS coefficient covered by CDF in the range of $[-60 \mathrm{dBsm}, 30 \mathrm{dBsm}]$ (Figure $7 \mathrm{~b}, \mathrm{~d}$ ). Maintaining a certain flying speed $(20 \mathrm{Ma})$, the higher is the flying altitude, the smaller is the slope of CDF curve and the more gentle is its distribution. Similarly, at the same flying altitude, the larger is the flying Mach number, the wider is the DRCS coefficient range covered by the CDF curve, and the trend of curve change will be more gentle. 


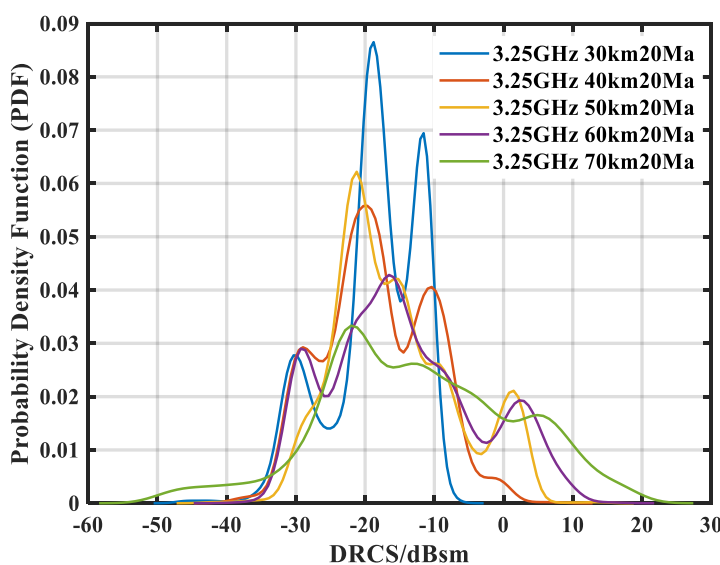

(a)

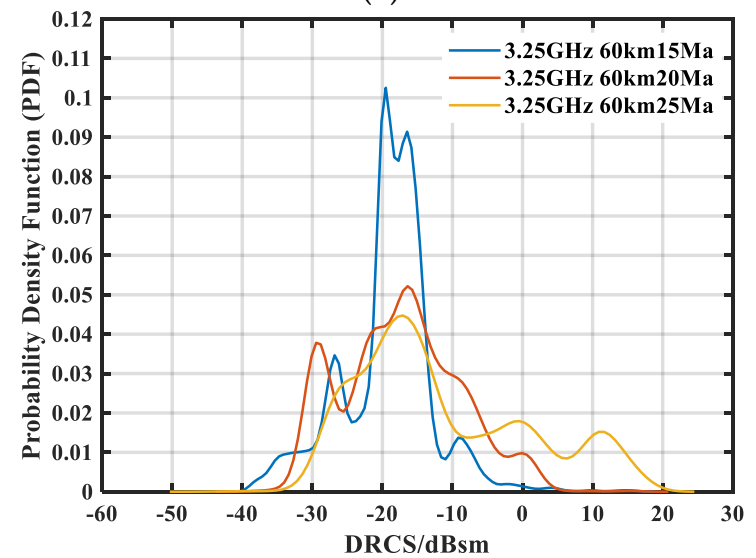

(c)

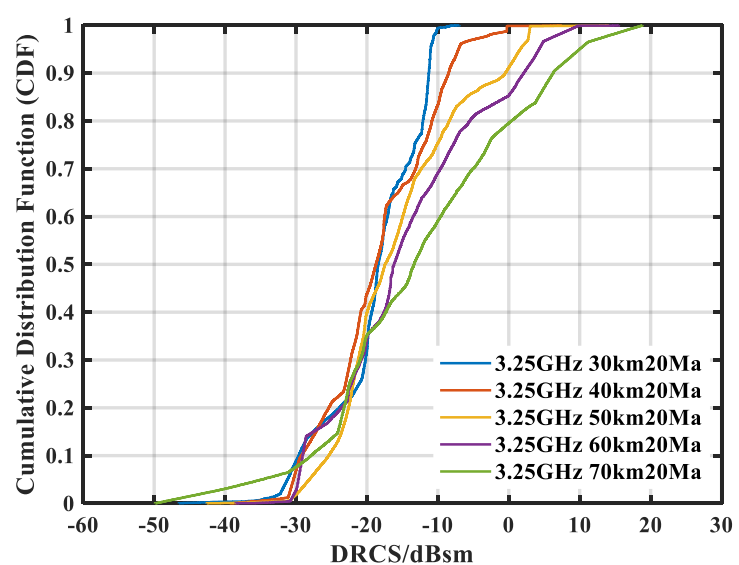

(b)

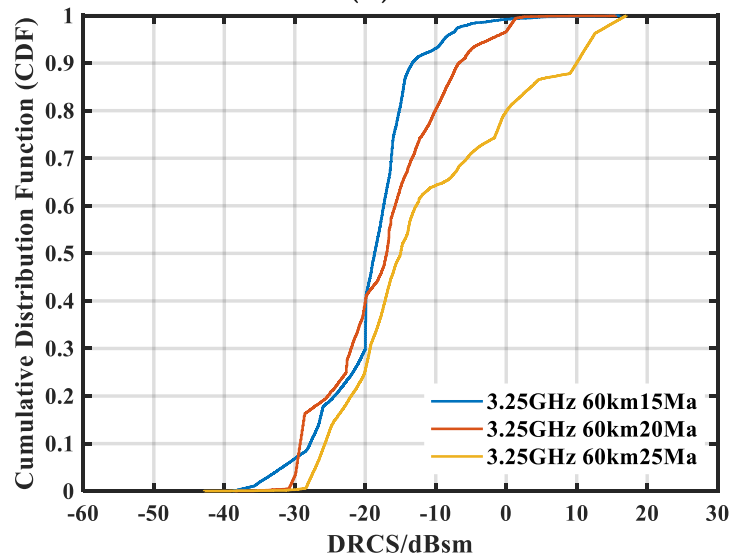

(d)

Figure 7. PDF and CDF distributions of DRCS time series for hypersonic vehicles under different flying conditions. Incident EM wave frequency $\mathrm{f}=3.25 \mathrm{GHz}$ : (a) PDF: $\mathrm{H}_{\mathrm{x}}=30 \mathrm{~km}, 40 \mathrm{~km}, 50 \mathrm{~km}, 60 \mathrm{~km}$ and $70 \mathrm{~km}, \mathrm{v}=20$ Mach; (b) CDF: $\mathrm{H}_{\mathrm{x}}=30 \mathrm{~km}, 40 \mathrm{~km}, 50 \mathrm{~km}, 60 \mathrm{~km}$ and $70 \mathrm{~km}, \mathrm{v}=20$ Mach; (c) PDF: $\mathrm{H}_{\mathrm{x}}=$ $60 \mathrm{~km}, \mathrm{v}=15$ Mach, 20 Mach, 25 Mach; and (d) CDF : $\mathrm{H}_{\mathrm{x}}=60 \mathrm{~km}, \mathrm{v}=15$ Mach, 20 Mach, 25 Mach.

\section{Feature Extraction of Dynamic RCS Time Series Based on Continuous Wavelet Transform}

\subsection{Continuous Wavelet Transform and Time-Scale Plane}

In this subsection, the dynamic RCS time series of the target spacecraft is extracted by using continuous wavelet transform (CWT) of the joint time-scale plane description signal, where the scale corresponds to the frequency. Based on the different frequency information, the processing of the dynamic RCS observation sequence of low-resolution space-borne radar can clearly show the details of the RCS observation sequence in the time domain and frequency domain, which would be more conducive to extracting the features of the hypersonic vehicle for different flying states.

Scale factor $a$ and time factor $b$ are discretized to realize CWT. The observation sequence of dynamic RCS of space-borne radar is set as $\operatorname{DRCS}(t), t=1,2, \ldots, T_{b}$, where $T_{b}$ is the total length of dynamic RCS observation data. Then, the wavelet transform of $\operatorname{DRCS}(\mathrm{t})$ is:

$$
\mathrm{W}_{\operatorname{DRCS}}(\mathrm{a}, \mathrm{b})=\sum_{\mathrm{t}=1}^{\mathrm{T}} \operatorname{DRCS}(\mathrm{t}) \Psi^{*}\left(\frac{\mathrm{t}-\mathrm{b}}{\mathrm{a}}\right)
$$

where $\mathrm{b}=1,2, \ldots, \mathrm{T}_{\mathrm{b}}$ and $\mathrm{a}=1,2, \ldots, \mathrm{S}_{\mathrm{a}}$.

To enable the final results to be compared for different flying states of the target spacecraft, during the flying process, the range of angle variation with which the space-borne radar illuminates the target spacecraft was always $2.5^{\circ} \leq \theta \leq 177.5^{\circ}$; thus, the total time $\mathrm{T}_{\mathrm{b}}$ in DRCS $(\mathrm{t})$ of the target 
spacecraft for different flying states was different. In the simulations, the time interval was $\mathrm{T}_{\mathrm{s}}=1 \mathrm{~s}$, the maximum value of scale factor was $S_{a}=600$ and the Symlet(symN) wavelet (compactly supported wavelets with least asymmetry and highest number of vanishing moments for a given support width) was used as the mother wavelet, where $\mathrm{N}$ was calculated as 4 . As shown in Figure $8 \mathrm{a}-\mathrm{d}$, when the flying altitude of spaceborne radar is $\mathrm{H}=200 \mathrm{~km}$, for four different flying states and incident EM wave frequencies, the time-scale diagram of dynamic RCS time series of the hypersonic vehicle is obtained after continuous wavelet transform.

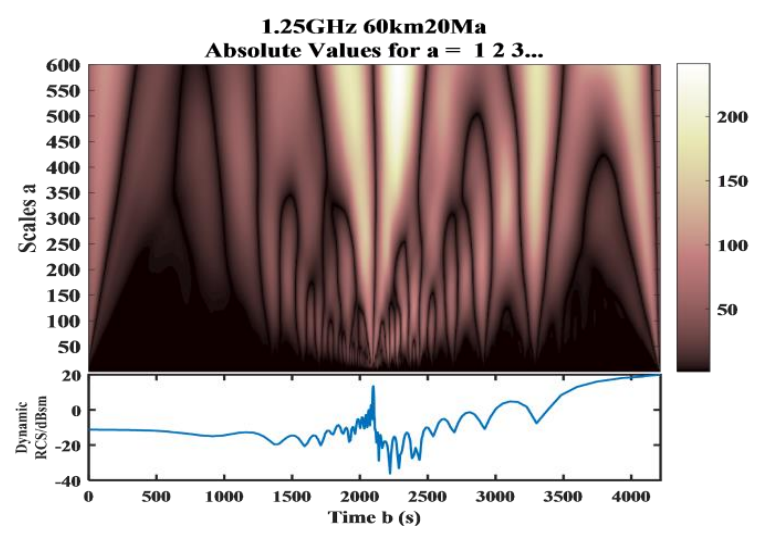

(a)

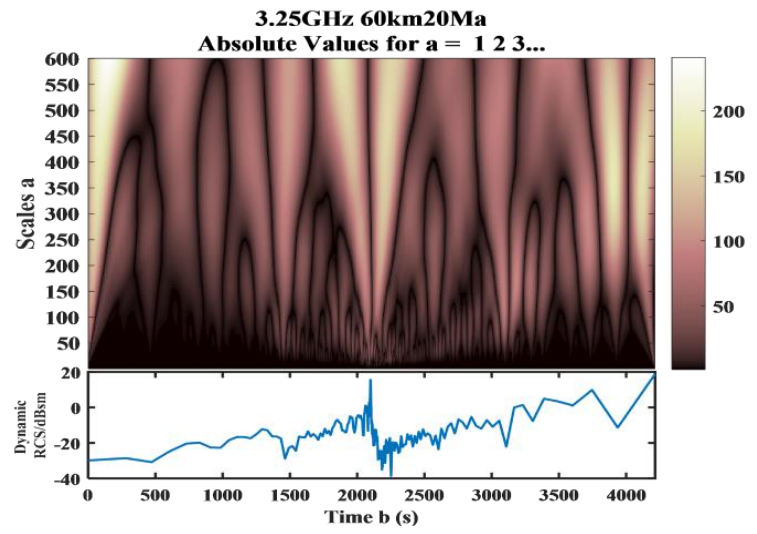

(c)

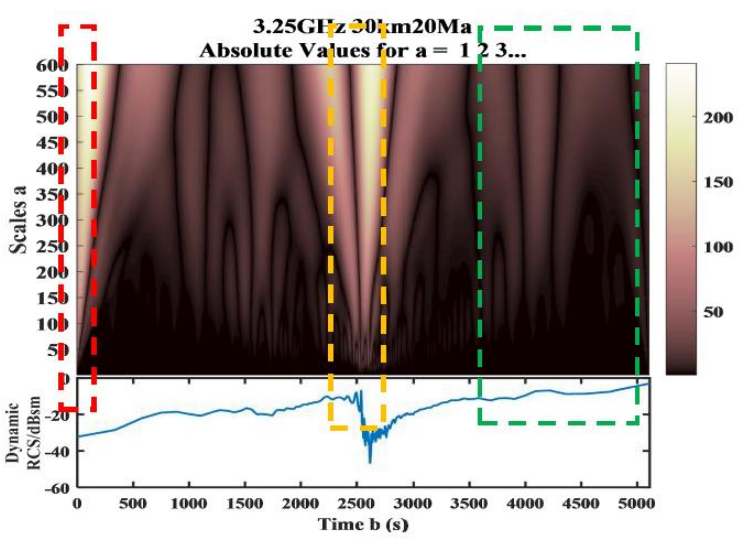

(b)

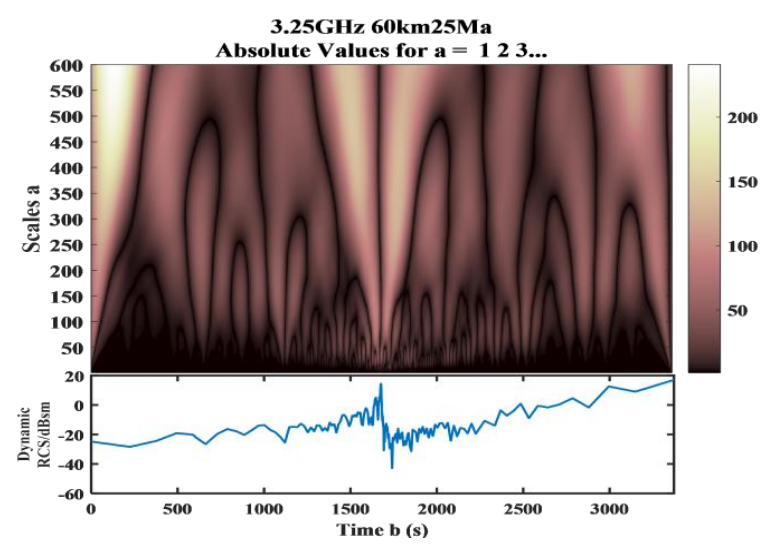

(d)

Figure 8. Time-scale diagram of dynamic RCS time series of a hypersonic vehicle covered with a plasma sheath after continuous wavelet transform at a velocity and height above the ground of: (a) 20 Mach and $60 \mathrm{~km}$, respectively (incident wave frequency is $1.25 \mathrm{GHz}$ ); (b) 20 Mach and $30 \mathrm{~km}$, respectively (incident wave frequency is $3.25 \mathrm{GHz}$ ); (c) 20 Mach and $60 \mathrm{~km}$, respectively (incident wave frequency is $3.25 \mathrm{GHz}$ ); and (d) $25 \mathrm{Mach}$ and $60 \mathrm{~km}$, respectively (incident wave frequency is $3.25 \mathrm{GHz}$ ).

Figure 8a shows the time-scale diagram of the target spacecraft at $60 \mathrm{~km}$ and $20 \mathrm{Mach}$. The abscissa is the time factor, indicating the total flying time of the target spacecraft, and the ordinate is the scale factor, representing the scale range of the wavelet transform. Here, the scale factor corresponds to the frequency, i.e., the smaller is the scale factor, the greater is the frequency, and vice versa. In other words, when the scale factor a is smaller, more wavelet functions are needed to fit the dynamic RCS time series in this time period, which can more accurately reflect the fluctuation details of the dynamic RCS sequence of the target spacecraft over time. The color intensity in Figure 8a represents the size of the absolute value of the wavelet coefficient, i.e., the brighter is the color, the greater is the value of the wavelet coefficient. For the dynamic RCS time series of the hypersonic vehicle, when the curve fluctuates violently and jitters and sudden changes occur, the inner product of the dynamic RCS function and the sym 4 wavelet function shows that the result value is the largest; that is, the value of the wavelet coefficient is the largest. In other words, the higher is the similarity between the target 
dynamic RCS time series and the sym4 wavelet of the same length in this period, the brighter is the representative part in the time-scale diagram.

Because the fluctuation change recorded by the wavelet function is 0 by default at the initial position, the initial value of the target dynamic RCS curve is less than 0. For example, in Figure 8b, the value of the dynamic RCS curve of the target spacecraft at the initial position is $-32 \mathrm{dBm}$; hence, the wavelet transform determines by default that the initial position of the dynamic RCS sequence of the target suddenly changes. In other words, it is shown in the time-scale diagram that relatively bright longitudinal stripes will appear in the initial time period, as shown in the area enclosed by a red dashed line in Figure 8b.

In addition, when the target spacecraft is flying in the area covered by the space-borne radar during the period of 2000-3000 s, the target dynamic RCS curve fluctuates violently, and the corresponding time-scale diagram shows bright longitudinal stripes, as shown in the area enclosed by the yellow dashed line in Figure 8b. During the period of 3000-5000 s, the dynamic RCS curve fluctuates gently. At the small scale, the wavelet coefficient is almost 0 , and the time-scale image is darker. Compared with the larger scale range, the dynamic RCS curve at this time exhibits weak fluctuation, the wavelet coefficient value is small and the time-scale image has low brightness, as shown in the area enclosed by the green dashed line in Figure 8b. In Figure 8c,d, the dynamic RCS curves of the target spacecraft under these two calculation conditions fluctuate wildly. Therefore, the time-scale image after wavelet transformation has relatively dense longitudinal bright stripes, which can clearly distinguish the characteristics of the target dynamic RCS curve for different flying states.

\subsection{Feature Extraction of Dynamic RCS on Time-Scale Plane}

Here, we set $A=\left|W_{\operatorname{DRCS}}(a, b)\right|$. Because the volume of data of Matrix A after wavelet transformation is very large with extensive information redundancy, it is obviously unrealistic to take it directly as a feature, which means we need to extract features with fewer stable dimensions from Matrix A. Because the dynamic RCS observation sequence of the target has random stability, it can be considered to extract mathematical statistical features from Matrix A. After many simulation experiments and using a method based on [39,40], we extracted 10 valid statistical features. They are the maximum singular value feature, mean value feature, maximum value feature, variance feature, scale barycenter feature, central moment feature and invariant moment feature, respectively. The specific calculation formulas appear in Appendix A. We can combine these 10 effective statistical features in a template for target recognition, which is expected to lay a solid foundation for the recognition of hypersonic vehicle in the future. Table 2 lists the 10 relatively stable statistical features extracted from the time-scale plane for different flying states and calculation conditions.

In addition, we examined whether the influence of the plasma sheath on the eigenvalues of the dynamic RCS sequence of a hypersonic vehicle could be more convincingly explained. Suppose that along the horizontal flying path of the target spacecraft, a pure metal blunt cone spacecraft model is placed at each unit angle incident position of space-borne radar. The radar illumination conditions are $\mathrm{H}=200 \mathrm{~km}$ and $\mathrm{f}=3.25 \mathrm{GHz}$. When the target spacecraft is at $60 \mathrm{~km}$ and $20 \mathrm{Ma}$, the change law of time as a function of angle is taken as the calculation condition to solve the dynamic RCS of the metallic blunt cone spacecraft. The ten effective eigenvalues mentioned above were extracted on the scale-time plane and compared with those extracted by the hypersonic vehicle covered with a plasma sheath at a flying altitude of $60 \mathrm{~km}$ and a speed of 20 Ma under the same radar incident conditions, as listed in Table 3.

The eigenvalue data shown in Tables 2 and 3 indicate that, for features $f_{1}, f_{3}$ and $f_{4}$, which differ from the other features, the eigenvalue of pure metal targets is larger than that of the other targets covered with a plasma sheath, which plays an important role in the classification and recognition of target spacecraft. At the same time, it also shows that the contributions of various original eigenvalues extracted from the dynamic RCS samples of the target spacecraft for classification are different, and the degree of importance of different eigenvalues to samples also varies. In addition, for hypersonic 
vehicles, the existence of a plasma sheath can greatly change the size of the ten effective statistical eigenvalues, by differentiating and changing them depending on the radar illumination conditions and flying states of the target spacecraft. These feature sets are expected to play an important role in the classification and recognition of hypersonic vehicles in near space.

Table 2. Ten effective statistical characteristic values extracted on the time-scale plane after continuous wavelet transform for the dynamic RCS of a hypersonic vehicle under different calculation conditions.

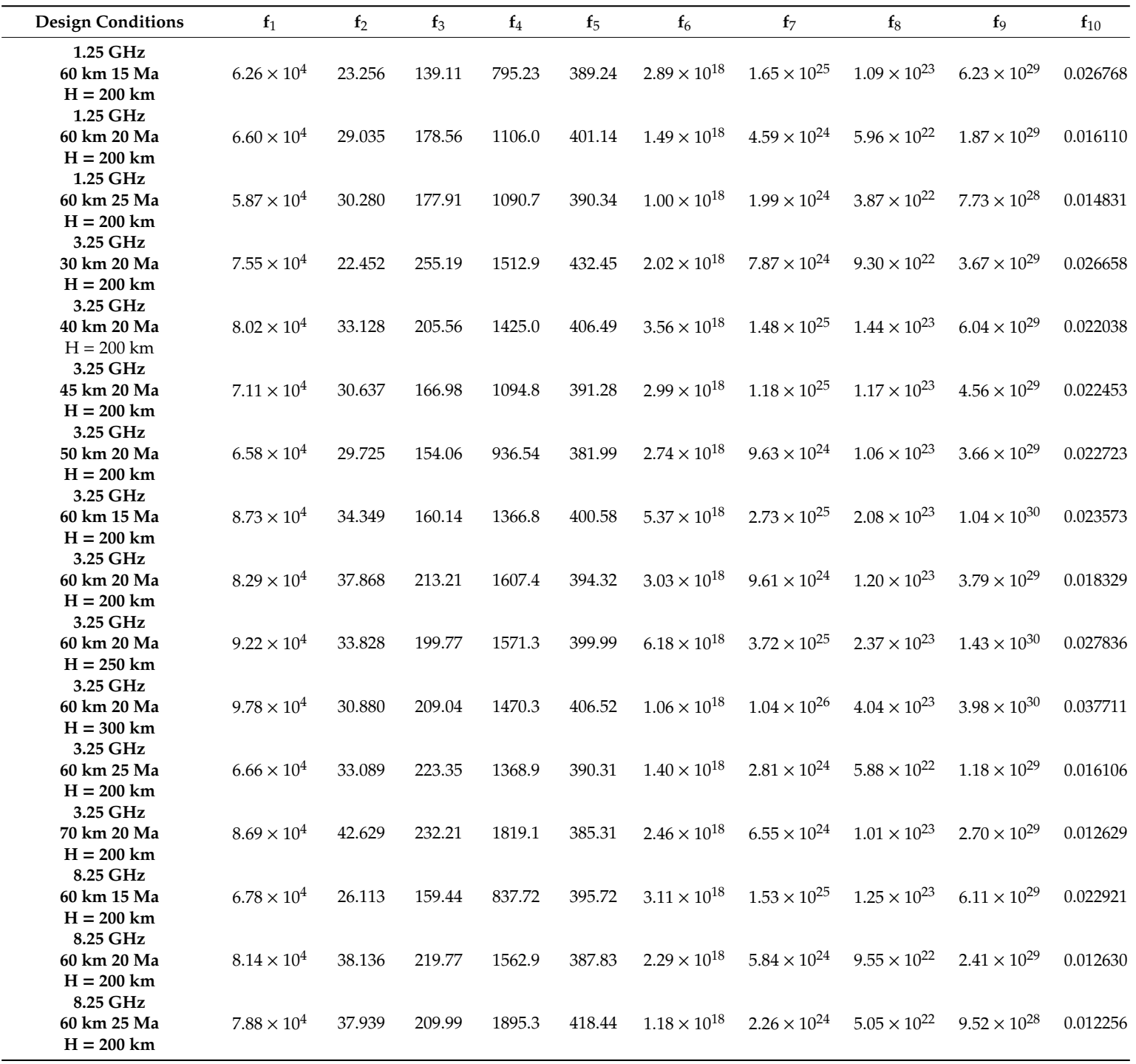

Table 3. Comparison of the effective statistical characteristic values depending on whether the hypersonic vehicle is covered with a plasma sheath $(\mathrm{f}=3.25 \mathrm{GHz}$ and $\mathrm{H}=200 \mathrm{~km}$ for the flying parameters $60 \mathrm{~km}$ and $20 \mathrm{Ma})$.

\begin{tabular}{|c|c|c|c|c|c|c|c|c|c|c|}
\hline $\begin{array}{c}\text { Design } \\
\text { Conditions }\end{array}$ & $\mathbf{f}_{1}$ & $\mathbf{f}_{2}$ & $\mathbf{f}_{3}$ & $\mathbf{f}_{4}$ & $\mathbf{f}_{5}$ & $\mathbf{f}_{6}$ & $\mathbf{f}_{7}$ & $\mathbf{f}_{8}$ & $\mathbf{f}_{9}$ & $\mathbf{f}_{10}$ \\
\hline $\begin{array}{c}\text { Metal Cone } \\
3.25 \mathrm{GHz} \\
60 \mathrm{~km} 20 \mathrm{Ma} \\
\mathrm{H}=200 \mathrm{~km} \\
3.25 \mathrm{GHz}\end{array}$ & $1.09 \times 10^{5}$ & 34.486 & 362.15 & 3895.5 & 401.67 & $2.08 \times 10^{18}$ & $1.07 \times 10^{25}$ & $8.64 \times 10^{22}$ & $4.30 \times 10^{29}$ & 0.015726 \\
\hline $\begin{array}{c}60 \mathrm{~km} 20 \mathrm{Ma} \\
\mathrm{H}=200 \mathrm{~km}\end{array}$ & $8.29 \times 10^{4}$ & 37.868 & 213.21 & 1607.4 & 394.32 & $3.03 \times 10^{18}$ & $9.61 \times 10^{24}$ & $1.20 \times 10^{23}$ & $3.79 \times 10^{29}$ & 0.018329 \\
\hline
\end{tabular}




\section{Conclusions}

In this paper, the parallel PO method based on the IZMM is used to simulate and analyze the dynamic EM scattering characteristics of the hypersonic vehicle covered with plasma sheath under the condition of controllable computing time. By using the angle-time conversion method in the side station level flying maneuver mode, we analyze the DRCS of a hypersonic target for different flying states and under different calculation conditions and establish a relatively complete simulation database. Then, the CWT is used to extract ten valid statistical characteristic values on the time-scale plane, which lays a data foundation for the target recognition of a hypersonic vehicle. The results of our calculations enable us to draw the following conclusions:

1. According to the EM scattering characteristics of hypersonic vehicle at different flying altitudes, we can find that the plasma sheath can greatly change the fluctuation trend of the BRCS of the hypersonic target, making its oscillation law irregular and difficult to predict. In the large illuminate angle range, the BRCS has a reduction effect, which is due to the collision absorption of the incident EM wave in the plasma environment with high electron density. In addition, when the hypersonic target is flying at different altitudes, the EM parameters of the plasma sheath coated on the surface of the hypersonic vehicle are also different, resulting in the BRCS of the vehicle extremely sensitive to the change of flying state, which is a great challenge for hypersonic target identification.

2. Based on the flying path along the horizontal trajectory, we calculated the DRCS of the hypersonic vehicle for different flying states, and the influence of flying altitude and velocity on the recognition of hypersonic target using DRCS is quantitatively analyzed by using mathematical sensitivity functions PDF and CDF. The results show that the frequency of the incident EM wave, detection altitude and polarizations of SBR, the flying altitude of the target spacecrafts and the flying speed all affect the DRCS time series of a hypersonic vehicle covered with a plasma sheath, but it is possible to detect and identify hypersonic vehicles. In addition, CWT is used to extract the characteristics of the DRCS sequence of the vehicle, and a relatively complete DRCS database for hypersonic vehicle recognition is established.

3. Finally, based on the urgent needs and existing bottlenecks of hypersonic targets recognition capability, the research results of this paper explore and reveal the dynamic EM scattering characteristics of plasma sheath flow field of hypersonic vehicle. By showing the mathematical and transformation characteristics of DRCS time series, the EM scattering characteristics of hypersonic vehicle covered with plasma sheath are effectively connected with the field of target recognition, and, as a key link, the technical methods are effectively applied to the actual research. The research work published in this paper provides theoretical analysis basis and sample identification database for hypersonic vehicle detection technology based on satellite platform and provides reliable data support and sample reference for target detection, imaging and recognition in hypersonic field in the future.

Author Contributions: Conceptualization, Z.B. and J.L.; methodology, Z.B.; formal analysis, Z.B.; investigation, J.L. and L.G.; resources, J.L. and L.G.; project administration, J.L. and L.G.; writing一original draft preparation, Z.B. and J.L.; and writing-review and editing, Z.B., J.L. and L.G. All authors have read and agreed to the published version of the manuscript.

Funding: This research was funded in part by the National Natural Science Foundation of China under Grants 61431010 and 61627901, in part by the Natural Science Foundation of Shaanxi Province of China under Grant 2019JM-206, in part by the Key Laboratory Foundation under Grant 6142502190203, in part by the National Key Laboratory Foundation under Grant HTKJ2019KL504014 and in part by the Foundation for Innovative Research Groups of the National Natural Science Foundation of China under Grant 61621005.

Conflicts of Interest: The authors declare no conflict of interest. 


\section{Appendix A}

Effective statistical features $f_{1}, f_{2} \ldots f_{10}$ for hypersonic target recognition are extracted from Matrix A, as shown below:

1. Maximum singular value feature of Matrix A.

If $\mathrm{A}$ is regarded as an $\mathrm{M} \times \mathrm{N}$ dimensional matrix, there exists unitary Matrices $\mathrm{U}$ and $\mathrm{V}$ of $\mathrm{M} \times \mathrm{M}$ and $\mathrm{N} \times \mathrm{N}$ dimensions, such that $\mathrm{A}=\mathrm{U \Sigma V}^{\mathrm{H}}$, where the superscript $\mathrm{H}$ represents the conjugate transpose of the matrix and $\Sigma$ is an $\mathrm{M} \times \mathrm{N}$ dimensional diagonal matrix, of which the elements on the main diagonal are non-negative and arranged in the following order:

$$
\sigma_{11} \geq \sigma_{22} \geq \ldots \geq \sigma_{\mathrm{hh}} \geq 0
$$

where the maximum singular value is:

$$
\mathrm{f}_{1}=\sigma_{11}
$$

2. Mean value of Matrix A

The mean value is:

$$
\mathrm{f}_{2}=\frac{1}{\mathrm{M} \times \mathrm{N}} \sum_{\mathrm{i}=1}^{\mathrm{M}} \sum_{\mathrm{j}=1}^{\mathrm{N}} \mathrm{A}(\mathrm{i}, \mathrm{j})
$$

3. Maximum characteristic of Matrix A

Extract the maximum value of the element in Matrix A as a valid statistical feature $f_{3}$.

4. Variance characteristics of Matrix A

The variance characteristics is:

$$
\mathrm{f}_{4}=\frac{1}{\mathrm{M} \times \mathrm{N}} \sum_{\mathrm{i}=1}^{\mathrm{M}} \sum_{\mathrm{j}=1}^{\mathrm{N}}\left[\mathrm{A}(\mathrm{i}, \mathrm{j})-\mathrm{f}_{2}\right]^{2}
$$

5. Scale barycenter feature

A is regarded as an $\mathrm{M} \times \mathrm{N}$ dimensional discrete two-dimensional digital image, which is a scale-time two-dimensional digital image. Then, the barycenter of scale is characterized as:

$$
f_{5}=\frac{\sum_{i=1}^{M} \sum_{j=1}^{N}[i \times A(i, j)]}{\sum_{i=1}^{M} \sum_{j=1}^{N} A(i, j)}
$$

6. Central moment feature

Let $\mathrm{c}=\frac{\sum_{\mathrm{i}=1}^{M} \sum_{j=1}^{N}[j \cdot \mathrm{A}(\mathrm{i}, \mathrm{j})]}{\sum_{\mathrm{i}=1}^{M} \sum_{j=1}^{N} \mathrm{~A}(\mathrm{i}, \mathrm{j})}$, and the central moment of the discrete two-dimensional digital image A is defined as:

$$
\mathrm{u}_{\mathrm{pq}}=\sum_{\mathrm{i}=1}^{\mathrm{M}} \sum_{\mathrm{j}=1}^{\mathrm{N}}\left[\left(\mathrm{i}-\mathrm{f}_{5}\right)^{\mathrm{p}}(\mathrm{j}-\mathrm{c})^{\mathrm{q}} \mathrm{A}(\mathrm{i}, \mathrm{j})\right]
$$

with respect to $(p, q)=(2,2),(2,4),(4,2)$ and $(4,4)$.

Finally, we obtain the central moment feature $\mathrm{f}_{6}=\mathrm{u}_{22}, \mathrm{f}_{7}=\mathrm{u}_{24}, \mathrm{f}_{8}=\mathrm{u}_{42}$ and $\mathrm{f}_{9}=\mathrm{u}_{44}$.

7. Invariant moment feature

The central moment is used to deduce the set of seven two-dimensional invariants of rotation, scaling and translation invariants, which can effectively represent the characteristics of time-scale two-dimensional digital images. 
The central moment of the normalized order $(p, q)$ is:

$$
\eta_{\mathrm{pq}}=\mathbf{u}_{\mathrm{pq}} / \mathbf{u}_{00}^{\gamma}
$$

where $\mathrm{p}, \mathrm{q}=0,1,2, \ldots, \gamma=(\mathrm{p}+\mathrm{q}) / 2+1$.

The seven invariant moments are:

$$
\begin{gathered}
M_{1}=\eta_{20}+\eta_{02} \\
M_{2}=\left(\eta_{20}-\eta_{02}\right)^{2}+4 \eta_{11}^{2} \\
M_{3}=\left(\eta_{30}-3 \eta_{12}\right)^{2}+\left(3 \eta_{21}-\eta_{03}\right)^{2} \\
M_{4}=\left(\eta_{30}+\eta_{12}\right)^{2}+\left(\eta_{21}+\eta_{03}\right)^{2} \\
M_{5}=\left(\eta_{30}-3 \eta_{12}\right)\left(\eta_{30}+\eta_{12}\right)\left[\left(\eta_{30}+\eta_{12}\right)^{2}-3\left(\eta_{21}+\eta_{03}\right)^{2}\right] \\
+\left(3 \eta_{21}-\eta_{03}\right)\left(\eta_{21}+\eta_{03}\right)\left[\left(3\left(\eta_{30}+\eta_{12}\right)^{2}-\left(\eta_{21}+\eta_{03}\right)^{2}\right]\right. \\
M_{6}=\left(\eta_{20}-\eta_{02}\right)\left[\left(\eta_{30}+\eta_{12}\right)^{2}-\left(\eta_{21}+\eta_{03}\right)^{2}\right] \\
+4 \eta_{11}\left(\eta_{30}+\eta_{12}\right)\left(\eta_{21}+\eta_{03}\right) \\
M_{7}=\left(3 \eta_{21}-\eta_{03}\right)\left(\eta_{30}+\eta_{12}\right)\left[\left(\eta_{30}+\eta_{12}\right)^{2}-3\left(\eta_{21}+\eta_{03}\right)^{2}\right] \\
+\left(3 \eta_{21}-\eta_{03}\right)\left(\eta_{21}+\eta_{03}\right)\left[\left(3\left(\eta_{30}+\eta_{12}\right)^{2}-\left(\eta_{21}+\eta_{03}\right)^{2}\right]\right.
\end{gathered}
$$

When extracting the features of the dynamic RCS time series of a hypersonic vehicle, the invariance of $\mathrm{M}_{1}$ is well maintained, and the seven invariants have approximately the same impact on classification. Therefore, $\mathrm{M}_{1}$ is selected as the characteristic parameter of the dynamic RCS of the target spacecraft, i.e., $\mathrm{f}_{10}=\mathrm{M}_{1}$.

\section{References}

1. Tao, S.F.; Fan, Z.H.; Liu, W.J.; Chen, R.S. Electromagnetic scattering analysis of a conductor coated by multilayer thin materials. IEEE Antennas Wirel. Propag. Lett. 2013, 12, 1033-1036. [CrossRef]

2. Bian, Z.; Li, J.T.; Guo, L.X.; Luo, X. Range profile analysis of hypersonic vehicles covered by inhomogeneous plasma sheath using physical optics. IEEE Trans. Plasma Sci. 2019, 47, 4961-4970. [CrossRef]

3. Rybak, J.P.; Churchill, R.J. Progress in reentry communications. IEEE Trans. Aerosp. Electron. Syst. 1971, 7, 879-894. [CrossRef]

4. Wang, Y.X.; Liu, S.; Zhong, S.Y. Studies on the effects of the plasma wake flow fields of hypersonic reentry blunt cone on electromagnetic wave. IEEE Trans. Plasma Sci. 2019, 47, 3991-3996. [CrossRef]

5. Yang, X.; Wei, B.; Yin, W. Analysis on the characteristics of EM waves propagation in the plasma sheath surrounding a hypersonic vehicle. IEEE Trans. Plasma Sci. 2017, 45, 2922-2928. [CrossRef]

6. Chen, J.; Tang, G.X.; Zhang, J.B.; Huo, L. Analysis of electromagnetic wave propagation in unmagnetized hypersonic vehicle plasma sheath using the variational method. IEEE Trans. Plasma Sci. 2019, 47, 5259-5263. [CrossRef]

7. Skolnik, M.I. Radar Handbook, 3rd ed.; Publishing House of Electronics Industry: Beijing, China, 2010; pp. 702-757.

8. Keidar, M.; Kim, M.; Boyd, I. Electromagnetic reduction of plasma density during atmospheric reentry and hypersonic flights. J. Spacecr. Rocket. 2008, 45, 445-453. [CrossRef]

9. Starkey, R.P. Hypersonic vehicle telemetry blackout analysis. J. Spacecr. Rocket. 2015, 52, 426-438. [CrossRef]

10. Ma, J.G.; Zhao, H.Z.; Fu, Q. Space target recognition algorithm based on RCS sequence. Aerosp. Electron. Warf. 2007, 23, 14-16.

11. Xin, Y.L.; Xu, R.C. Methods of target recognition based on low-resolution radar. Mod. Electron. Tech. 2005, 19, $17-22$.

12. Jones, W.L.; Cross, A.E. Electrostatic Probe Measurements of Plasma Parameters for Two Reentry Flight Experiments at 25000 Feet per Second; Technical Report; NASA: Washington, DC, USA, 1972; p. 6617. 
13. Swift, C.T.; Beck, F.B.; Thomson, J.; Castellow, S.L. RAM C-III S-band diagnostic experiment. In Proceedings of the NASA SP-252 4th Plasma Sheath Symp; NASA: Washington, DC, USA, 1971; pp. 137-155.

14. Luebbers, R.J.; Hunsberger, F.; Kunz, K.S. A frequency-dependent finite-difference time-domain formulation for transient propagation in plasma. IEEE Trans. Antennas Propag. 1991, 39, 29-34. [CrossRef]

15. Wei, H.L.; Liu, Y.M.; Shi, L.; Yao, B.; Li, X.P. A 3-D total-field/scattered-field plane-wave source for the FDTD analysis of re-entry plasma sheath. IEEE Trans. Antennas Propag. 2020, 68, 6214-6225. [CrossRef]

16. Lim, Y.; Seo, B.; Nam, S. FDTD simulation of three-wave scattering process in time-varyinig cold plasma sheath. IEEE Access 2019, 7, 106713-106720. [CrossRef]

17. Zhang, Y.; Liu, Y.M.; Li, X.P. A 2-D FDTD model for analysis of plane wave propagation through the reentry plasma sheath. IEEE Trans. Antennas Propag. 2017, 65, 5940-5948. [CrossRef]

18. Wang, H.; Xu, L.; Li, B.; Descombes, S.; Lanteri, S. An exponential-based DGTD method for modeling 3-D plasma-surrounded hypersonic vehicles. IEEE Trans. Antennas Propag. 2020, 68, 3847-3858. [CrossRef]

19. Qian, J.W.; Zhang, H.L.; Xia, M.Y. Modelling of electromagnetic scattering by a hypersonic cone-like body in near space. Int. J. Antennas Propag. 2017, 2017, 11. [CrossRef]

20. Sha, Y.X.; Zhang, H.L.; Guo, X.Y.; Xia, M.Y. Analyses of electromagnetic properties of a hypersonic object with plasma sheath. IEEE Trans. Antennas Propag. 2019, 67, 2470-2481. [CrossRef]

21. Liu, S.H.; Guo, L.X.; Pan, W.T.; Chen, W.; Xiao, Y.F. PO calculation for reduction in radar cross section of hypersonic targets using RAM. Phys. Plasmas 2018, 25, 062105. [CrossRef]

22. Liu, S.H.; Guo, L.X. Analyzing the electromagnetic scattering characteristics for 3-D inhomogeneous plasma sheath based on PO method. IEEE Trans. Plasma Sci. 2016, 44, 2838-2843. [CrossRef]

23. Chang, Y.; Chen, W.F.; Luo, N.; Wu, Q.F. Analysis of the spatial scattering characteristic for the reentry target cloaked by plasma based on the physical optics method. J. Microw. 2008, 24, 1-6.

24. Bian, Z.; Li, J.T.; Guo, L.X.; Luo, X. Analyzing the electromagnetic scattering characteristics of a hypersonic vehicle based on the inhomogeneity zonal medium model. IEEE Trans. Antennas Propag. 2020. [CrossRef]

25. Jia, J.S.; Liang, Z.C.; He, H.F. Field-discrete Monte Carlo simulation of electromagnetic scattering from plasma sheath. Guid. Fuze 2018, 39, 43-47.

26. Liu, W.M.; Tong, C.M.; Wang, T.; Zhai, X.Y. Analysis of the impact of aircraft movement feature on dynamic RCS series. Fire Control Command Control 2017, 42, 33-38.

27. Zhou, C.; Li, Z.; Xu, C. Research on space target size identification based on narrow band RCS. Radio Eng. 2018, 48, 859-864.

28. Guay, R.; Drolet, G.; Bray, J.R. Measurement and modelling of the dynamic radar cross-section of an unmanned aerial vehicle. IET Radar Sonar Navig. 2017, 11, 1155-1160. [CrossRef]

29. Liu, J.; Fang, N.; Wang, B.F.; Xie, Y.J. A novel dynamic RCS simulation and analysis method considering attitude perturbation. J. Electromagn. Waves Appl. 2015, 29, 1841-1858. [CrossRef]

30. Zhou, C.; Zhang, X.K.; Zhang, J.W.; Zhang, C.X. Analysis of dynamic RCS time series of typical stealth aircraft. J. Air Force Eng. Univ. Nat. Sci. Ed. 2013, 14, 15-18.

31. Walter, M.; Shutin, D.; Fiebig, U. Delay-Dependent Doppler Probability Density Functions for Vehicle-to-Vehicle Scatter Channels. IEEE Trans. Antennas Propag. 2014, 62, 2238-2249. [CrossRef]

32. Ouchi, K. A theory on the distribution function of backscatter radar cross section from ocean waves of individual wavelength. IEEE Trans. Geosci. Remote Sens. 2000, 38, 811-822. [CrossRef]

33. Zyweck, A.; Bogner, R.E. Radar target classfication of commercial aircraft. IEEE Trans. Aerosp. Electron. Syst. 1996, 32, 598-606. [CrossRef]

34. Nie, X.C.; Yuan, N.; Li, L.W.; Gan, Y.B. Fast Analysis of RCS Over a Frequency Band Using Pre-Corrected FFT/AIM and Asymptotic Waveform Evaluation Technique. IEEE Trans. Antennas Propag. 2008, 56, 3526-3533. [CrossRef]

35. Wang, T.; Bi, W.J.; Zhao, Y.L.; Xue, W.C. Radar target recognition algorithm based on RCS observation sequence-Set-valued identification method. J. Syst. Sci. Complex. 2016, 29, 573-588. [CrossRef]

36. Lv, Y.G.; Guo, L.X.; Li, J.T. Hypersonic Vehicle Plasma Sheath and Electromagnetic Characteristics Data Manual, 1st ed.; Science Press: Beijing, China, 2019; pp. 33-60.

37. Ginzburg, V.L. The Propagation of Electromagnetig Waves in Plasmas, 2nd ed.; Pergamon, Science Press: Beijing, China, 1970; p. 2168.

38. Liu, Z.W.; Bao, W.M.; Li, X.P.; Liu, D.L. A segmentation calculation method for plasma collision frequency considering the electro-magnetic wave driving effect. Acta Phys. Sin. 2014, 63, 235201. 
39. Ma, J.G.; Li, B.G.; Wang, Y.M.; Zhao, H.Z. Space target recognition algorithm of low resolution radar based on discrete wavelet translation. Signal Process. 2006, 22, 70-72. (In Chinese)

40. Li, X. Research on Radar Target Feature Identification Based on RCS Sequence. Master's Thesis, Beijing Jiaotong University, Beijing, China, 2018.

(C) 2020 by the authors. Licensee MDPI, Basel, Switzerland. This article is an open access article distributed under the terms and conditions of the Creative Commons Attribution (CC BY) license (http://creativecommons.org/licenses/by/4.0/). 